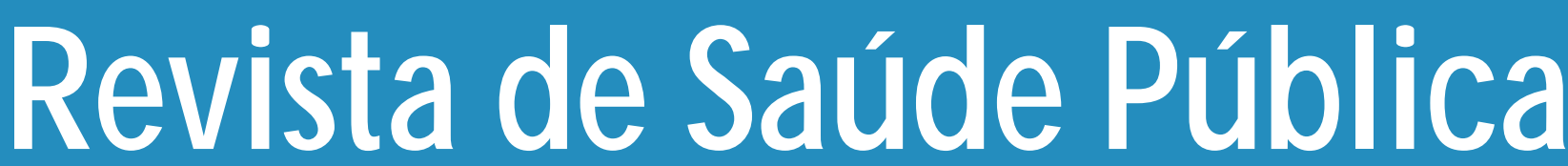

Journal of Public Health

\title{
Leishmaniose tegumentar americana no Estado do Acre, Brasil
}

American cutaneous leishmaniasis in the State of Acre, Brazil

\section{Natal S da Silva, Araguacy B Viana, José A Cordeiro e Carlos E Cavasini}

Departamento de Doenças Infecciosas e Parasitárias da Faculdade de M edicina de São José do Rio Preto. São José do Rio Preto, SP - Brasil (NSS, JAC, CEC), Universidade Federal de São Carlos. São Carlos, SP - Brasil $(A B V)$

SILVA N atal S, Araguacy B Viana, José A Cordeiro e Carlos E Cavasini Leishmaniose tegumentar americana no Estado do Acre, Brasil Rev. Saúde Pública, 33 (6):554-59, 1999 www.fsp.usp.br/rsp 


\title{
Leishmaniose tegumentar americana no Estado do Acre, Brasil*
}

\section{American cutaneous leishmaniasis in the State of Acre, Brazil}

\author{
Natal S da Silva, Araguacy B Viana, José A Cordeiro e Carlos E Cavasini \\ Departamento de Doenças Infecciosas e Parasitárias da Faculdade de Medicina de São José do Rio \\ Preto. São José do Rio Preto, SP - Brasil (NSS, JAC, CEC), Universidade Federal de São Carlos. São \\ Carlos, SP - Brasil (ABV)
}

\section{Descritores}

Leishmaniose mucocutânea, epidemiologia. Levantamentos epidemiológicos. Distribuição espacial.

\section{Keywords}

Leishmaniasis, mucocutaneous epidemiology. Health surveys. Residence characteristics.

\section{Resumo}

\section{Objetivo}

Apresentar levantamento estatístico descritivo da leishmaniose tegumentar americana (LTA) no Estado do Acre.

\section{Métodos}

Os dados obtidos foram extraídos dos formulários da "Campanha Contra a Leishmaniose", no período de janeiro de 1992 a dezembro de 1997. As variáveis estudadas foram submetidas a análise estatística descritiva.

\section{Resultados}

O total de casos registrados foi de 2.557. Foi adotada a divisão do Acre em meso e microrregiões para apresentação dos resultados. A maior prevalência foi na microrregião de Brasiléia (231,8 casos/10.000 hab.). A forma clínica predominante foi cutânea (84,05\%). A maior ocorrência foi no sexo masculino (71,02\%). Portadores com idade de até 24 anos corresponderam a 50\% dos casos. Há uma predominância nas ocupações rurais. O exame clínico foi usado para diagnosticar 83,97\% dos casos. A maior média de tempo de espera para procurar tratamento médico foi registrada na mesorregião do Vale do Juruá (10,37 meses).

\section{Conclusão}

Os altos índices de LTA cutâneo e cutâneo-mucosa encontrados sugerem a necessidade de serem feitas pesquisas sobre os reflexos psicossociais e para identificar fatores que influem na demora do tratamento dos casos.

\footnotetext{
Abstract

Objective

Present a statistical survey of American Cutaneous Leishmaniasis (ACL) in the State of Acre.

Methods

Data were obtained from the forms of the "Campaign against Leishmaniasis", from January 1992 to December 1997. Descriptive statistical analysis was applied. Results

There were 2.557 registered cases. The highest prevalence was found at the microregion of Brasiléia (231.8 cases/10,000 inhab.). The predominant clinical form was cutaneous (84.05\%). The disease occurred mostly among males (71.02\%). Half of the cases were among people with 24 years of age or younger. Most cases were people
}

Correspondência para/Correspondence to: Carlos Eugênio Cavasini

Avenida Faria Lima, 5416

15090-000 São José do Rio Preto, SP - Brasil

E-mail: natalss@ hotmail.com
*Trabalho realizado nos Departamentos: Epidemiologia e Saúde Coletiva e de Doenças Infecciosas e Parasitárias da Faculdade de Medicina de São José do Rio Preto. Edição subvencionada pela Fapesp (Processo n. 98/13915-5). Recebido em 17.11.1998. Aprovado em 8.6.1999. 
with rural occupations. $83.97 \%$ of the cases were diagnosed by clinical examination. The longest period to seek medical treatment was registered in the mesoregion of Juruá Valley (10.37 months)

Conclusion

The high number of cases suggests that it might be necessary to study the psychosocial implications of the disease and identify factors contributing to the delay in treatment.

\section{INTRODUÇÃO}

No Brasil, a prevalência da leishmaniose tegumentar americana (LTA) vem aumentando consideravelmente. Na década de 1980, foram registrados pelo Ministério da Saúde 131.166 casos $^{5}$. Lacerda (1994) ${ }^{8}$ demonstrou que somente nos anos de 1990 e 1991 foram notificados no Brasil por esse ministério 25.929 casos, sendo 15.083 somente na região Norte. Fato esse que denota a importância epidemiológica de realizar um estudo estatístico da LTA no contexto amazônico e em particular no Estado do Acre, o qual carece de estudos científicos acerca desta endemia.

O presente trabalho tem por objetivo realizar um estudo descritivo da LTA no Estado do Acre.

\section{MÉTODOS}

O Estado do Acre está localizado no sudoeste da Amazônia brasileira, na Amazônia Ocidental, região Norte. Possui uma área de $152.588 \mathrm{~km}^{2}$ e faz limite com a Bolívia, Peru e com os Estados do Amazonas e Rondônia (Figura 1). O clima é equatorial, a hidrografia faz parte da Bacia Amazônica. A vegetação é caracterizada por floresta tropical úmida e o relevo é formado por largas planícies com forma plana e moderadamente ondulada. Sua população está estimada em 500.185 habitantes $^{11}$.

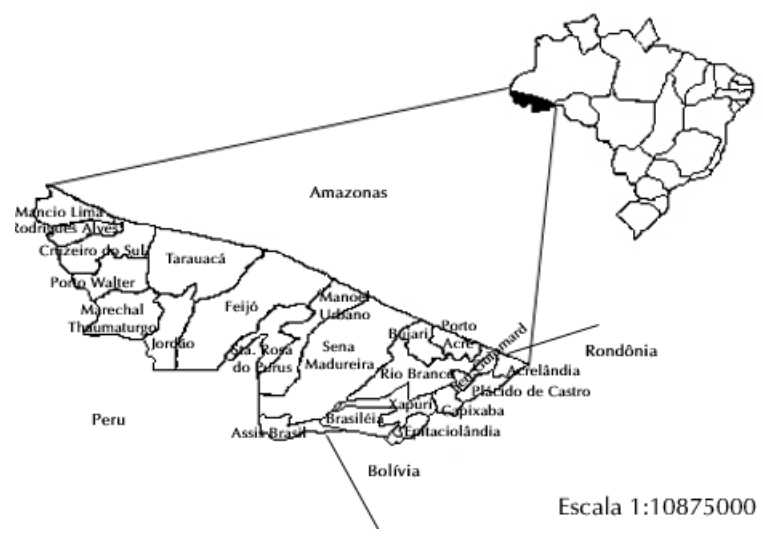

Figura 1 - Localização e divisão política do Estado do Acre.

O Acre está divido em duas mesorregiões (Vale do Juruá e Vale do Acre) e cinco microrregiões (Cruzeiro do Sul, Tarauacá, Sena Madureira, Rio Branco e
Brasiléia) (IBGE) ${ }^{4,11}$. O Vale do Juruá, com 141.165 habitantes no ano de $1997^{4}$, é formado pelas microrregiões de Cruzeiro do Sul e de Tarauacá. A primeira é composta pelos municípios de Cruzeiro do Sul, Mâncio Lima, Marechal Thaumaturgo, Porto Walter e Rodrigues Alves. A segunda, pelos municípios de Feijó, Jordão e Tarauacá ${ }^{11}$. As microrregiões de Sena Madureira, Rio Branco e Brasiléia formam o Vale do Acre, o qual possuía 359.020 habitantes no ano de $1997^{4}$. Sena Madureira é composta pelos municípios de Sena Madureira, Santa Rosa do Purus e Manoel Urbano. Rio Branco, pelos municípios de Rio Branco, Acrelândia, Bujari, Capixaba, Plácido de Castro, Porto Acre e Senador Guiomard. A microrregião de Brasiléia, pelos municípios de Brasiléia, Assis Brasil, Epitaciolândia e Xapuri ${ }^{11}$.

Até 1 de janeiro de 1993, o Estado contava com apenas 12 municípios, quando foram criados os municípios de Acrelândia, Bujari, Capixaba, Epitaciolândia, Jordão, Marechal Thaumaturgo, Porto Acre, Porto Walter, Rodrigues Alves e Santa Rosa do Purus. Totalizam atualmente 22 municípios ${ }^{4,11}$.

Os dados para a realização do presente trabalho foram coletados na Coordenação Regional do Acre da Fundação Nacional de Saúde (FNS), órgão do Ministério da Saúde responsável pelo cadastro de todas as notificações dos casos de LTA no Estado. Foram utilizados os formulários da "Campanha Contra a Leishmaniose - Registro dos Casos Novos e Importados de Leishmaniose Visceral e Tegumentar" no período de janeiro de 1992 a dezembro de 1997, com 2.557 casos registrados. Nos formulários constam os dados: idade, sexo, localidade, tipo de ocupação dos pacientes e início da doença, data da procura para tratamento, métodos de diagnóstico e formas clínicas diagnosticadas. Esses dados foram submetidos à análise estatística descritiva.

Tendo em vista a não-confiabilidade do levantamento populacional do Acre nos anos de 1992, 1993,1994 e 1995, empregou-se o método de interpolação linear para fazer uma estimativa populacional nos referidos anos. Com o objetivo de obter um resultado mais fidedigno, estimou-se a população do Estado no meio dos respectivos anos em estudo, para efeito de cálculo da prevalência da LTA. 
Para estudar melhor a distribuição da LTA, as ocupações dos pacientes foram agrupadas em três categorias: rural (como seringueiro, peão, diarista, pecuarista e índio), não rural (militar, funcionário público, médico, dentista, enfermeiro, e outras) e não determinadas, cujas ocupações não se caracterizam como rural ou não rural (doméstica, aposentado, menor e estudante).

A LTA foi também avaliada com relação ao número de casos novos, formas clínicas, sexo e idade dos pacientes, métodos de diagnóstico e tempo de procura para tratamento médico.

\section{RESULTADOS}

Dos 2.557 registros de LTA, encontraram-se 2.316 casos novos no Estado, dos quais 85,2\% foram locados na mesorregião do Vale do Acre. A microrregião de Rio Branco contribuiu com maior percentual de casos novos no período $(41,1 \%)$. A microrregião de Sena Madureira foi a única que mostrou uma diminuição progressiva no número de casos novos no período e contínua redução da prevalência nos anos de 1993 (38 casos/10.000 hab.), 1994 (13,4 casos/10.000 hab.), 1995 (10 casos/10.000 hab.) e 1996 (2,7 casos/10.000 hab.).

Em 1996, a microrregião de Brasiléia apresentou significativo percentual de casos novos $(45,0 \%)$ e uma maior prevalência (59,8 casos/10.000 hab.), tendo também a maior prevalência dentro do período ( 231,8 casos $/ 10.000$ hab.). O Município de Xapuri mostrou uma prevalência de 501,7 casos por 10.000 habitantes no período e 129,4 no ano de 1996, contribuindo significativamente com o percentual de sua microrregião (Tabela 1).
A microrregião de Cruzeiro do Sul destacou-se por apresentar menor prevalência no período ( 25,8 casos/ 10.000 hab.). A mesorregião do Vale do Acre apresentou maior prevalência no período (60,2 casos/10.000 hab.), quando comparada com a do Vale do Juruá (30,3 casos/ 10.000 hab.). Verificou-se também que a prevalência no Estado foi de 55,7 casos/10.000 habitantes no período.

Foram diagnosticadas três formas clínicas no Estado: cutânea, mucosa e cutâneo-mucosa. A cutânea apresentou o maior percentual $(84,1 \%)$, e a cutâneomucosa o menor percentual $(4,73 \%)$ dos casos registrados no Estado no período. A microrregião de Tarauacá diferenciou-se das demais com uma maior ocorrência da forma mucosa $(41,0 \%)$ e da forma cutâneo-mucosa $(19,1 \%)$, quando comparada com as demais microrregiões (Tabela 2).

Nos municípios de Jordão e Porto Walter, 100\% dos casos foram da forma cutânea. Em Capixaba, Mâncio Lima, Plácido de Castro, Porto Acre, Senador Guiomard e Xapuri, esse índice ficou acima de $90 \%$. Feijó obteve o maior percentual de mucosa (50,3\%). Já Manoel Urbano apresentou maior índice de cutâneo-mucosa (59,4\%). O município de Marechal Thaumaturgo apresentou significativa ocorrência da forma cutâneomucosa $(23,1 \%)$, e o município de Assis Brasil, um expressivo percentual da forma mucosa $(33,7 \%)$.

Quanto ao sexo, observou-se maior ocorrência de LTA no sexo masculino $(71,0 \%)$ no Estado. Contudo, houve um aumento crescente no percentual do número de casos em mulheres nos anos de $1992(23,8 \%)$, 1993 (25,04\%), 1994 (31,9\%) e 1995 (35,7\%), e uma redução nos anos de $1996(31,06 \%)$ e 1997 (27,6\%).

Tabela 1 - Prevalência da LTA por 10.000 habitantes, segundo microrregiões, mesorregiões e Estado do Acre.

\begin{tabular}{lrrrrrrr}
\hline Região & 1992 & 1993 & 1994 & 1995 & 1996 & 1997 & 1992 a 1997 \\
\hline Cruzeiro do Sul & 5,4 & 3,4 & 2,8 & 5,2 & 5,1 & 4,1 & 25,8 \\
Tarauacá & 8,5 & 4,9 & 7,8 & 4,5 & 5,9 & 6,6 & 38,0 \\
Sena Madureira & 13,2 & 38,0 & 13,4 & 10,0 & 2,7 & 3,6 & 80,4 \\
Rio Branco & 6,3 & 9,5 & 5,7 & 6,4 & 6,3 & 6,5 & 40,6 \\
Brasiléia & 32,8 & 42,6 & 33,2 & 25,4 & 59,8 & 37,4 & 231,8 \\
\hline Vale do Juruá & 6,5 & 3,9 & 4,5 & 5,0 & 6,4 & 5,0 & 30,3 \\
Vale do Acre & 10,0 & 16,1 & 9,6 & 8,9 & 12,0 & 9,7 & 60,2 \\
\hline Estado & 9,1 & 12,3 & 8,1 & 7,8 & 10,1 & 8,4 & 55,7 \\
\hline
\end{tabular}

LTA - Leishmaniose tegumentar americana

Tabela 2 - Distribuição das formas clínicas da LTA, segundo microrregiões, mesorregiões e Estado do Acre, no período de 1992 a 1997.

\begin{tabular}{lrrrrrrr}
\hline Região & \multicolumn{1}{l}{$\mathrm{C}^{*}$} & \multicolumn{2}{c}{$\mathrm{M}^{* *}$} & \multicolumn{3}{c}{$\mathrm{CM}^{* * *}$} & Total \\
& $\mathrm{N}$ & $\%$ & $\mathrm{~N}$ & $\%$ & $\mathrm{~N}$ & $\%$ & $\mathrm{~N}$ \\
\hline Cruzeiro do Sul & 176 & 81,9 & 17 & 7,9 & 22 & 10,2 & 215 \\
Tarauacá & 73 & 39,9 & 75 & 41,0 & 35 & 19,1 & 183 \\
Sena Madureira & 191 & 79,3 & 23 & 9,6 & 27 & 11,2 & 241 \\
Rio Branco & 941 & 89,6 & 83 & 7,9 & 26 & 2,5 & 1.050 \\
Brasiléia & 768 & 88,5 & 89 & 10,3 & 11 & 1,3 & 868 \\
\hline Vale do Juruá & 249 & 62,6 & 92 & 23,1 & 57 & 14,3 & 398 \\
Vale do Acre & 1.900 & 87,8 & 195 & 9,0 & 64 & 3,2 & 2.159 \\
\hline Estado & 2.149 & 84,1 & 287 & 11,2 & 121 & 4,7 & 2.557 \\
\hline
\end{tabular}

* Cutânea

** Mucosa

*** Cutâneo-mucosa 
Quanto à idade, 25\% dos casos foram registrados em crianças até 15 anos (primeiro quartil, Q1, igual a 15 anos), $50 \%$ em pessoas até 24 anos, com mediana (Md) igual a 24 anos; coeficiente de variação (n) igual a 63,9\%; desvio-padrão (s) de 17,5; e média aritmética $(\mu)$ igual a 27,4 anos. A microrregião de Sena Madureira apresentou 50\% dos casos na população com idade de até 20 anos ( $\mathrm{Md}=20)$ e Cruzeiro do Sul até $28(\mathrm{Md}=28)$. Quanto às microrregiões de Brasiléia e Sena Madureira, 25\% dos casos ocorreram em crianças com idade de até 12 anos $(\mathrm{Q} 1=12)$. As distribuições das idades entre o primeiro e o terceiro quartis de Brasiléia (Q1=12 anos, Q3=33 anos) e de Rio Branco (Q1=17 anos, Q3=39 anos) são razoavelmente simétricas quando observadas próximo de suas medianas. O coeficiente de variação demonstra que a microrregião de Cruzeiro do Sul obteve o menor coeficiente de variação $(n=54,7 \%$; $\mathrm{s}=16,77 ; \mu=30,64$ anos), enquanto a de Brasiléia apresentou o maior ( $\mathrm{n}=69,9 \%$; $\mathrm{s}=17,42 ; \mu=24,91$ anos).

Quanto à ocupação dos portadores, no Estado a zoonose foi mais freqüente em populações com ocupações rurais $(40,2 \%)$. Fazendo-se esta mesma análise nas microrregiões, verificou-se que a de Rio Branco apresentou menor índice em populações com ocupação rural $(33,3 \%)$ e um entre as não rurais $(24,9 \%)$. $\mathrm{Na}$ microrregião de Brasiléia o maior percentual foi de ocupações rurais $(49,2 \%)$, e o menor para as não rurais $(6,0 \%)$. Entretanto, a de Sena Madureira obteve uma maior notificação da doença entre as populações com ocupações não determinadas $(50,8 \%)$ (Tabela 3). Dentro destas últimas populações, as domésticas foram as que apresentaram maior índice de ocorrência no Estado (10,7\%).

No que se refere a métodos de diagnóstico, no Estado do Acre há um predomínio pelo clínico, feito através das manifestações clínicas da doença (84,0\%). $\mathrm{O}$ mesmo se reflete nas meso e microrregiões. No entanto, o Vale do Juruá apresentou o maior percentual de diagnósticos feitos por exame microscópico $(28,1 \%)$, quando comparado com o do Vale do Acre (6,4\%). Ao comparar as microrregiões, a de Cruzeiro do Sul apresentou maior percentual de diagnósticos feitos por exame microscópico $(47,9 \%)$ e a de Tarauacá por reação intradérmica de Montenegro (31,7\%).

Verificando-se o tempo transcorrido entre o início dos sintomas e o do tratamento, observou-se que no Vale do Juruá $50 \%$ dos pacientes levaram de 2 (Q1=2,03) a 9 meses $(\mathrm{Q} 3=9,43)$ para procurar tratamento, enquanto no Vale do Acre esse intervalo oscilou de 1 $(\mathrm{Q} 1=1,03$ meses) a 6 meses $(\mathrm{Q} 2=6,07$ meses). A mesorregião do Vale do Acre obteve maior coeficiente de variação relativa ( $n=262,5 \%$; $s=20,11 ; \mu=7,66$ meses) quando comparado com o Vale do Juruá ( $\mathrm{n}=205,5 \%$; $\mathrm{s}=21,31 ; \mu=10,37$ meses).

Na microrregião de Rio Branco, 50\% das pessoas atingidas levaram de $1(\mathrm{Q} 1=1,03$ meses) a 5 meses (Q3=5,07 meses) para procurar tratamento médico, enquanto na de Sena Madureira, 50\% dos pacientes levaram de 2 ( $\mathrm{Q} 1=2,03$ meses) a 13 meses (Q3=13,17 meses). Em todas as microrregiões houve uma considerável assimetria do primeiro e do terceiro quartis em torno da mediana (Figura 2).

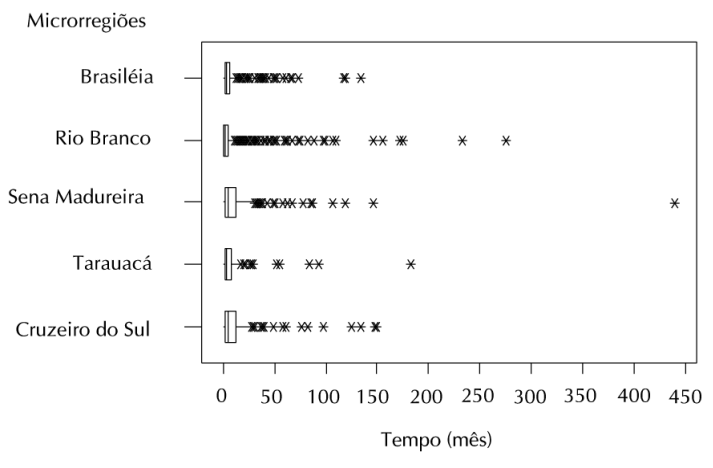

Figura 2 - Distribuição do tempo de espera dos portadores de LTA para procurar por tratamento médico, segundo microrregiões do Estado do Acre, no período de 1992 a 1997.

Tabela 3 - Distribuição das ocupações dos portadores de LTA, segundo microrregiões, mesorregiões e Estado do Acre, no período de 1992 a 1997.

\begin{tabular}{lrrrrrrr}
\hline Região & \multicolumn{2}{c}{$\mathrm{R}^{*}$} & \multicolumn{2}{c}{$\mathrm{NR}^{* *}$} & \multicolumn{2}{c}{$\mathrm{ND}^{* * *}$} & \multicolumn{2}{c}{ Total } \\
& $\mathrm{N}$ & $\%$ & $\mathrm{~N}$ & $\%$ & $\mathrm{~N}$ & $\%$ & $\mathrm{~N}$ \\
\hline Cruzeiro do Sul & 85 & 39,4 & 35 & 16,2 & 96 & 44,4 & 216 \\
Tarauacá & 76 & 41,5 & 24 & 13,1 & 83 & 45,4 & 183 \\
Sena Madureira & 92 & 38,0 & 27 & 11,2 & 123 & 50,8 & 242 \\
Rio Branco & 348 & 33,3 & 261 & 25,0 & 437 & 41,8 & 1.046 \\
Brasiléia & 428 & 49,2 & 52 & 6,0 & 390 & 44,8 & 870 \\
Vale do Juruá & 161 & 40,4 & 59 & 14,8 & 179 & 44,9 & 399 \\
Vale do Acre & 868 & 40,2 & 340 & 15,8 & 950 & 44,0 & 2.158 \\
\hline Estado & 1.029 & 40,2 & 399 & 15,8 & 1.129 & 44,2 & 2.557 \\
\hline
\end{tabular}

* Ocupações rurais (seringueiro, agricultor, peão, diarista, pecuarista, índio)

** Ocupações não rurais (militar, funcionário público, médico, dentista, enfermeiro, motorista, operador de máquinas, vigilante)

*** Ocupações não determinadas como rurais ou não rurais (doméstica, aposentado, menor, estudante) 
Brasiléia apresentou o menor coeficiente de variação ( $\mathrm{n}=186,2 \%$; $\mathrm{s}=11,73 ; \mu=6,3$ meses), enquanto Rio Branco foi o maior ( $n=283,1 \%$; $s=21,29 ; \mu=7,52$ meses). A Figura 2 mostra que em todas as microrregiões ocorreram valores atípicos; destacam-se, para procurar tratamento médico, um paciente em Sena Madureira com tempo de espera de 36,5 anos e outro, em Rio Branco, com 26 anos de espera.

\section{DISCUSSÃO}

Quanto ao número de casos novos por mesorregiões, verificou-se que a grande maioria deles foi locada no Vale do Acre. Contudo, deve-se levar em conta que essa mesorregião possui um número de habitantes muito maior do que a do Vale do Juruá. Todavia, o estudo da prevalência não deixa dúvidas de que no Vale do Acre a LTA constitui uma endemia de maior relevância epidemiológica que na outra mesorregião (Tabela 1). Esse fato ocorre principalmente porque a microrregião de Brasiléia contribuiu para elevar o número de casos novos e a prevalência de sua mesorregião.

O Acre não figura entre os principais focos amazônicos de LTA, havendo maior destaque para os Estados do Pará, Amazonas e Rondônia ${ }^{5,6,7,8,13}$. Lacerda $^{8}$ (1994) sugere que o Estado do Acre tenha respondido pela segunda menor ocorrência de LTA na região Norte do Brasil, no ano de 1991, a qual representou apenas $1,7 \%$ das ocorrências no mesmo ano no país. O que parece denotar que o Acre também não é uma das mais importantes zonas endêmicas no contexto brasileiro. Isso não diminui, entretanto, a necessidade de um programa de controle epidemiológico dessa zoonose, pois no contexto do Estado há importantes áreas endêmicas, como o Município de Xapuri, o qual apresentou no período em estudo uma alta prevalência.

Quanto às formas clínicas da LTA, os resultados obtidos estão de acordo com os realizados por outros autores em outros Estados, como Maranhão e Bahia ${ }^{2}$, Pará $^{7}$, São Paulo ${ }^{10}$ e Amazonas ${ }^{13}$, os quais também encontraram uma maior ocorrência da forma cutânea em seus estudos.

Os altos índices de LTA cutânea e cutâneo-mucosa encontrados nos municípios de Feijó, Manoel Urbano, Marechal Thaumaturgo e Assis Brasil sugerem, devido aos seus prováveis efeitos mutilantes, maior atenção aos reflexos psicossociais que essas deformações provocam. Esse fato sugere a realização de futuras pesquisas enfocando esse aspecto.

O estudo quanto ao sexo mostra que no Acre a distribuição da LTA está de acordo com trabalhos realiza- dos em outros Estados brasileiros ${ }^{2,7}$, sobressaindose, portanto, um maior percentual de pacientes do sexo masculino acometidos pela doença.

Quanto ao estudo da faixa etária dos pacientes, os resultados obtidos com o coeficiente de variação no Acre ( $n=63,4 \% ; s=17,5 ; \mu=27,4$ anos) denotam grande variabilidade na idade dos portadores da doença em todo o Estado. A média de idade (27,4 anos) aproximase do estudo feito por Zoia et al. ${ }^{15}$ (1996) no Município de Porto Ferreira, em São Paulo, ( $\mu=32$ anos). Observase também que os resultados da ocorrência em crianças no Acre se assemelham com os valores obtidos por Stolf et al. ${ }^{12}$ (1993), no Município de Itaporanga, em São Paulo, que encontrou 35\% de ocorrência de LTA em crianças menores de 12 anos durante um surto no referido município.

Ao analisar a variabilidade relativa entre as microrregiões, nota-se que não houve grandes oscilações, visto que o menor coeficiente de variação registrado foi de $54,7 \%$ na microrregião de Cruzeiro do Sul, e o maior foi de $69,9 \%$ na de Brasiléia. Porém, em todas as microrregiões, mesorregiões e no Estado, ocorreu uma considerável dispersão relativa das idades em relação às suas respectivas médias aritméticas.

Os valores encontrados no presente trabalho para a distribuição da LTA por ocupação concordam com estudos recentes ${ }^{2}$ feitos em outras regiões brasileiras, os quais também encontraram maior ocorrência da doença entre as populações com ocupações rurais. Esses valores estão de acordo com achados de outros autores $^{7}$ que afirmam ser essa zoonose uma doença ocupacional na Amazônia brasileira. Contudo, a leishmaniose tegumentar americana não figura entre as doenças que são conhecidas legalmente como ocupacionais, o que acarreta prejuízos aos trabalhadores doentes, se comparados aos benefícios que os mesmos teriam se a doença fosse assim considerada.

Os índices de pessoas com ocupação doméstica contaminadas no Estado e de populações não rurais na microrregião de Rio Branco sugerem que a doença na Amazônia acomete também pessoas com atividades que não exigem a entrada na floresta, o que pode reforçar os estudos de Falqueto et al. ${ }^{3}$ (1997), que relatam a possibilidade de contaminação no peridomicílio na periferia de Manaus, Amazonas. Situações similares vêm ocorrendo em outras regiões brasileiras ${ }^{14}$. Esses estudos enfatizam a variação na epidemiologia da LTA e seus vetores, observadas por Arias e Naiff ${ }^{1}$ (1981), na área periurbana de Manaus, onde o homem destruiu florestas e construiu conjuntos habitacionais em precárias condições sanitárias ao lado da mata. 
Em relação aos métodos de diagnóstico da LTA no Acre, o predomínio dos exames clínicos não compromete a confiabilidade dos resultados, visto que esse método possui uma margem de erro pequena, especialmente se o paciente procede de áreas endêmicas ou esteve em contato com as florestas de zonas leishmanióticas ${ }^{13}$. A utilização de técnicas mais modernas e mais confiáveis para o diagnóstico da doença, como a reação de polimerase em cadeia (PCR), esbarram no alto custo operacional atual, inviabilizando sua utilização rotineira em laboratórios de saúde pública ${ }^{7}$. Essa, provavelmente, é uma das causas de no Estado do Acre não serem utilizados outros exames laboratoriais além da microscopia e da reação intradérmica de Montenegro, os quais, de acordo com Falqueto et al. ${ }^{3}$ (1997), possuem uma sensibilidade de $80 \%$ a $97 \%$ para o primeiro e, aproximadamente, $80 \%$ para o segundo, com um baixo custo operacional.

\section{REFERÊNCIAS}

1. Arias IR, Naiff RD. The principal reservoir host of cutaneous leishmaniasis in the urbans areas of Manaus, Central Amazon of Brazil. Mem Inst Oswaldo Cruz 1981;76:279-86.

2. Costa JML, Balby ITA, Rocha EJSR, Silva AR, Rebêlo JMM, Ferreira LA et al. Estudo comparativo da leishmaniose tegumentar americana em crianças e adolescentes procedentes das áreas endêmicas de Buriticupu (Maranhão) e Corte de Pedra (Bahia), Brasil. Rev Soc Bras Med Trop 1998;31:279-88.

3. Falqueto A, Sessa PA. Leishmaniose tegumentar americana. In: Veronesi R. Tratado de infectologia. São Paulo: Atheneu; 1997. p. 1221-30.

4. Fundação IBGE. Divisão de Pesquisa do Estado do Acre (DIPEQ/AC). Anuário estatístico do Acre 1997. Acre; 1997.

5. Genaro O. Leishmaniose tegumentar americana. in: Neves D. Parasitologia humana. Rio de Janeiro: Atheneu; 1997. p. 41-60.

6. Habib F. Leishmanioses tegumentares. In: Linhares AC, compilador. Saúde na Amazônia. s.l: Associação Nacional de Programação Econômica e Social; 1983. p. 37-40.

7. Instituto Evandro Chagas. Relatório qüinqüenal 1991/ 1995. Belém: Fundação Nacional de Saúde/Ministério da Saúde; 1996.

8. Lacerda MM. The brazilian leishmaniasis control program. Mem Inst Oswaldo Cruz 1994;89:489-95.

9. Lainson R, Shaw JJ, Silveira FT, Souza AAA, Braga RR, Ishikawa EAY. Leishmanioses. In: Veronesi R. Tratado de infectologia. São Paulo: Atheneu; 1997. p.1495-7.
Em relação ao tempo transcorrido entre o início dos sintomas e início do tratamento, a média de 10,37 meses encontrada na mesorregião do Vale do Juruá, com uma considerável dispersão relativa dos dados próximo da média, sugere haver uma semelhança com o estudo do Instituto Evandro Chagas, no Estado do Pará 7 , o qual encontrou dois pacientes com história de 20 anos de evolução da doença sem a devida terapêutica. Uma das prováveis causas para essa demora, sugerida por Lainson et al. ${ }^{9}$ (1997), é "que os pacientes preferem ficar sem tratamento a percorrer grandes distâncias em prejuízo do trabalho e da família, tendo em vista que o mal atinge mais as classes pobres das áreas rurais da Amazônia”.

A constatação da demora para procurar tratamento médico sugere a realização de estudos posteriores para verificar a influência de fatores sociais, culturais, econômicos e geográficos, o que pode ser crucial para um provável controle epidemiológico da doença no Estado.

10. Rey L. Parasitologia: parasitos e doenças parasitárias do homem nas Américas e na África. 2a . ed. Rio de Janeiro: Guanabara Koogan; 1991. O complexo "leishmania braziliensis" e a leishmaniose tegumentar americana; p.193-201.

11. Rodrigues EB, Brito Filho AS, Nascimento MLM, Oliveira EC, Paula JVC, Lima RM et al. Estatística 1996 Acre. Rio Branco: Secretaria de Estado de Planejamento do Estado do Acre; 1997.

12. Stolf HO, Marques SA, Marques MEA, Yoshida ELA, Dillon NL. Surto de leishmaniose tegumentar americana em Itaporanga, São Paulo (Brasil). Rev Inst Med Trop São Paulo 1993;35:437-42.

13. Talhari S, Arias JR, Cunha MGS, Naiff RD, Naiff MF, Freitas RA et al. Leishmaniose no Estado do Amazonas, aspectos epidemiológicos, clínicos e terapêuticos. An Bras Dermatol 1988;63:433-8.

14. Teodoro U, La Salvia Filho V, Lima EM, Spinosa RP, Barbosa OC, Ferreira MEMC et al. Observações sobre o comportamento de flebotomíneos em ecótopos florestais e extraflorestais, em áreas endêmicas de leismaniose tegumentar americana, no norte do Estado do Paraná, sul do Brasil. Rev Saúde Pública 1993;27:242-9.

15. Zoia EN, Palombo AE, Pedrazzani ES. A leishmaniose no município de Porto Ferreira. Rev Bras Enferm 1996;49:445-58. 